\title{
A real world multicenter retrospective study on extramedullary disease from Balkan Myeloma Study Group and Barcelona University: analysis of parameters that improve outcome
}

\begin{abstract}
Meral Beksac, ${ }^{1}$ Guldane Cengiz Seval, ${ }^{1}$ Nicholas Kanellias, ${ }^{2}$ Daniel Coriu, ${ }^{3}$ Laura Rosiñol, ${ }^{4}$ Gulsum Ozet, ${ }^{5}$ Vesselina Goranova-Marinova, ${ }^{6}$ Ali Unal, ${ }^{7}$ Jelena Bila, ${ }^{8}$ Hayri Ozsan, ${ }^{9}$ Arben Ivanaj, ${ }^{10}$ Lejla Ibricevic Balić, ${ }^{11}$ Efstathios Kastritis, ${ }^{2}$ Joan Bladé, ${ }^{4}$ Meletios Athanasios Dimopoulos ${ }^{2}$

${ }^{1}$ Department of Hematology, School of Medicine, Ankara University, Ankara, Turkey; ${ }^{2}$ Department of Clinical Therapeutics, National and Kapodistrian University of Athens, School of Medicine, Athens, Greece; 'University of Medicine and Pharmacy "Carol Davila", Fundeni Clinical Institute, Bucharest, Romania; ${ }^{4}$ Hospital Clinic, IDIBAPS, Barcelona, Spain; ${ }^{5}$ Clinic of Hematology, Ankara Numune Education and

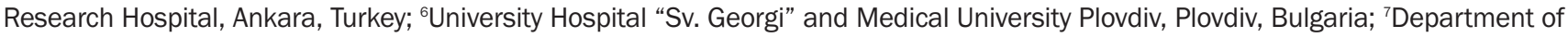
Hematology, School of Medicine, Erciyes University, Kayseri, Turkey; ${ }^{8}$ Faculty of Medicine, University of Belgrade, Belgrade, Serbia;

${ }^{9}$ Department of Hematology, School of Medicine, Dokuz Eylül University, Izmir, Turkey; ${ }^{10}$ University of Medicine Tirana, Tirana, Albania and ${ }^{11}$ Clinical Center of Sarajevo University, Sarajevo, Bosnia and Herzegovina
\end{abstract}

Published in Haematologica 2020;105(1):201-208.

doi:10.3324/haematol.2020.278272

(C)2021 Ferrata Storti Foundation

We have noticed an error in the progression-free survival of patients with extramedullary plasmacytoma in our article published in Haematologica in January 2020 (doi: HAEMATOL/2019/219295).

The following sentence in the abstract:

"Extramedullary plasmacytoma at relapse had the worst prognosis with a PFS of 13.6 months and overall survival of 11.4 months."

Should be replaced by:

"Extramedullary plasmacytoma at relapse had the worst prognosis with a PFS of 9.1 months and overall survival of 11.4 months."

Likewise, on page 205, the following sentence:

"However, if diagnosed at relapse, PFS and OS were 13.6 months and 11.4 months for EMP compared to 20.9 months $(P=0.249)$ and 39.8 months $(P=0.093)$ for $P O$, respectively (Table 2 and Figure 1$). "$

\section{Should be replaced by:}

"However, if diagnosed at relapse, PFS and OS were 9.1 months and 11.4 months for EMP compared to 20.9 months $(P=0.249)$ and 39.8 months $(P=0.093)$ for $P O$, respectively (Table 2 and Figure 1$). "$

The error was also present in Table 2.

The corrected Table 2 is shown below.

Table 2. Comparison of response, survival outcomes of extramedullary plasmacytomas (EMP) or paraosseous (P0) patients either at diagnosis or at relapse.

CR $(\%) \quad$ PFS (mos) OS (mos)

EMP

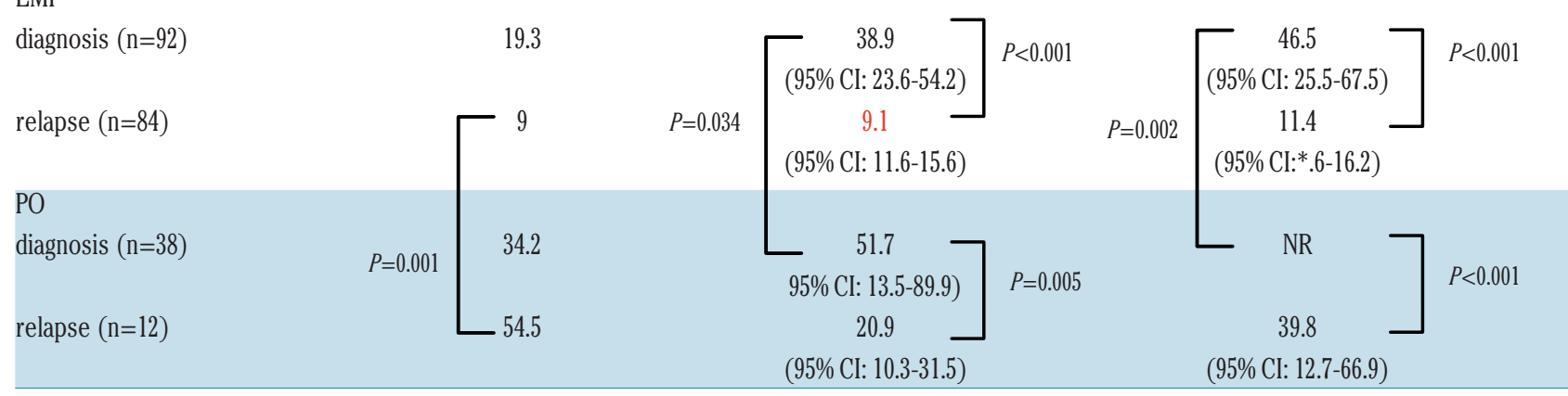

CR: complete remission; PFS: progression-free survival; OS: overall survival; n: number. 